\title{
ON TOTAL NONNORMING SUBSPACES
}

\author{
WILLIAM J. DAVIS AND JORAM LINDENSTRAUSS
}

\begin{abstract}
A Banach space $X$ has a total nonnorming subspace in its dual if and only if $X$ has infinite codimension in its second dual.
\end{abstract}

Let $X$ be a Banach space. A closed subspace $M$ of $X^{*}$ is said to be total if for every $0 \neq x \in X$ there is an $f \in M$ such that $f(x) \neq 0$. The subspace $M$ is said to be norming if $\|x\| \|=\sup \{|f(x)|: f \in M,\|f\|=1\}$ is equivalent to the original norm on $X$. Clearly every norming subspace of $X^{*}$ is total. The question "For which $X$ does $X^{*}$ have a total nonnorming subspace?" was raised by Dixmier [2]. It is well known that if $X$ is quasireflexive (i.e., $\operatorname{dim} X^{* *} / X<\infty$ ) then every total subspace of $X^{*}$ is norming (cf. Petunin [4] and Singer [7]).

We prove here the converse, i.e., that for every non-quasi-reflexive space $X$ there is a total nonnorming subspace in $X^{*}$. Strong partial results in this direction are already known (cf. Petunin [4]).

Let us first establish some definitions; we shall use in the following: A sequence $\left(x_{n}\right)$ in a Banach space $X$ is called a basis if for every $u \in X$ there is a unique sequence $\left(a_{n}\right)$ of scalars such that $u=\sum a_{n} x_{n}$ (the series converging in norm). A sequence $\left(z_{n}\right) \subset X$ is a basic sequence if it is a basis for $\left[z_{n}\right]$ (= closed linear span of $\left(z_{n}\right)$ ). The basis constant for $\left(z_{n}\right)$ is the smallest $K$ such that $\left\|\sum_{i=1}^{m} a_{i} z_{i}\right\| \leqq K\left\|\sum_{i=1}^{m+n} a_{i} z_{i}\right\|$ for all $\left(a_{i}\right), m, n>0$.

We begin with two lemmas, the first of which establishes the criterion we use in the main result.

Lemma 1. Let $X$ be a Banach space. Assume that $X$ has a closed infinite-dimensional subspace $Y$ and that $X^{* *}$ has a closed infinite-dimensional subspace $Z$ so that $Z \cap\left(Y^{* *}+X\right)=\{0\}$. Then $X^{*}$ contains a total nonnorming subspace. (We assume that $X$ and $Y^{* *}$ are embedded canonically in $X^{* *}$.)

Proof. There is no loss in generality to assume that $Y$ is separable. Let $\left\{y_{i}^{*}\right\}_{i=1}^{\infty}$ be unit vectors in $Y^{*}$ which are total over $Y$. Let $\left\{z_{i}\right\}_{i=1}^{\infty}$

Received by the editors February 16, 1971.

AMS 1970 subject classifications. Primary 46B10, 46B15.

Key words and phrases. Norming, quasi-reflexive space, dual space, conjugate space.

(C) American Mathematical Society 1972 
be a normalized basic sequence in $Z$. Define $T: Y^{* *} \rightarrow Z$ by $T y^{* *}=$ $\sum_{i=1}^{\infty} \epsilon_{i} y^{* *}\left(y_{i}^{*}\right) z_{i}$ where $\epsilon_{i}>0, \quad \sum \epsilon_{i} \leqq 1 / 2$. Clearly $T$ is compact, $\|T\| \leqq 1 / 2$ and $T_{\mid X}$ is one to one. Let $U=\left\{y^{* *}+T y^{* *} ; y^{* *} \in Y^{* *}\right\}$. It is easy to verify that the unit cell of $U$ and hence, by Krein's theorem, also $U$ itself is $w^{*}$ closed in $X^{* *}$. Let $M$ be the subspace $U^{\perp}$ of $X^{*}$. Since by our assumption $U \cap X=\{0\}$ it follows that $M$ is total. $M$ is however, nonnorming. Indeed, let $\epsilon>0$, and let $y \in Y$ be such that $\|y\|=1$ and $\|T y\| \leqq \epsilon$. For every $f \in M$ we have $f(y+T y)=0$ and hence $|f(y)|=$ $|f(T y)| \leqq \epsilon\|f\|$.

Lemma 2. If $X$ is not reflexive, $X$ contains an infinite-dimensional subspace $Y$ such that $X / Y$ is not reflexive.

Proof. By Singer [6] and Pelczynski [5] $X \supset\left(x_{n}\right)$, a basic sequence with $\left\|x_{n}\right\| \geqq 1$ for all $n$ such that $\left(\sum_{1}^{p} x_{n}\right)$ is bounded (in $p$ ). Let $Y=$ [ $x_{2 n-1}$ ] and $\varphi$ be the quotient map of $X$ onto $X / Y$. Then, if $K$ is the basis constant for $\left(x_{n}\right)$, it is well known that $\left\|\varphi\left(x_{2 n}\right)\right\| \geqq 1 / 2 K$. Further, $\left(\varphi\left(x_{2 n}\right)\right)$ is basic and $\sum_{1}^{p} \varphi\left(x_{2 n}\right)=\varphi\left(\sum_{1}^{2 p} x_{n}\right)$ is bounded. A basic sequence such as $\left(\varphi\left(x_{2 n}\right)\right)$ cannot exist in a reflexive space [6], so $X / Y$ is not reflexive.

Theorem. Let $X$ be a Banach space with $\operatorname{dim} X^{* *} / X=\infty$. Then $X^{*}$ contains a total nonnorming subspace.

Proof. By Lemma 1, it suffices to find an infinite-dimensional subspace $Y$ of $X$ such that $\operatorname{dim} X^{* *} /\left(X+Y^{* *}\right)=\infty$. (It follows, for example, as in [3], that there is a subspace $Z$ of $X^{* *}$ of infinite dimension such that $Z \cap\left(X+Y^{* *}\right)=\{0\}$.) For this, we may as well also assume that $X$ has no infinite-dimensional reflexive subspace (for such a subspace could take the role of $Y$ in Lemma 1). Now, using Lemma 2, construct a chain of subspaces

$$
X=X_{1} \supset X_{2} \supset X_{3} \supset X_{4} \supset \cdots
$$

such that for each $k, X_{k} \mid X_{k+1}$ is nonreflexive. Next, for $k=1,2, \cdots$, let $y_{k} \in X_{k} \sim X_{k+1}$ and set $Y=\left[y_{k}\right]$. To see that $Y$ satisfies the hypothesis of Lemma 1, notice first that $Y^{* *}=\left[y_{1}, y_{2}, \cdots, y_{k}\right]+\left(X_{k} \cap Y\right)^{* *}$. Thus, for each $k, X+Y^{* *}=X+\left(X_{k} \cap Y\right)^{* *} \subset X+X_{k}^{* *}$. By [1, Theorem 4.1], $X^{* *} /\left(X+X_{k}^{* *}\right)$ is isomorphic to $\left(\left(X / X_{k}\right)^{* *} /\left(X / X_{k}\right)\right)$ so that $X+Y^{* *}$ has codimension $\geqq k$ for every $k$, proving the theorem.

Remark. We use implicitly the fact [1, Theorem 4.1] that $X+Y^{* *}$ is always closed in $X^{* *}$. We have also used [1, Corollary 4.2] to see that $\operatorname{dim}\left(\left(X \mid X_{k}\right)^{*} /\left(X \mid X_{k}\right)\right) \geqq k$ for each $k$.

One interesting corollary of the theorem is the following stronger version of Lemma 2. "If $X$ is non-quasi-reflexive, then $X$ contains an 
infinite-dimensional subspace $Y$ such that $X / Y$ is non-quasi-reflexive." If $X$ contains an infinite-dimensional reflexive subspace $R$, then $X / R$ is the desired factor. Otherwise, let $Y$ be the subspace constructed in the proof of the theorem.

\section{REFERENCES}

1. P. Civin and B. Yood, Quasi-reflexive spaces, Proc. Amer. Math. Soc. 8 (1957), 906-911. MR 19, 756.

2. J. Dixmier, Sur un théorème de Banach, Duke Math. J. 15 (1948), 1057-1071. MR 10, 306.

3. V. I. Gurariĭ and M. I. Kadec, Minimal systems and quasi-complements in Banach space, Dokl. Akad. Nauk SSSR 145 (1962), 256-258 = Soviet Math. Dokl. 3 (1962), 966-968. MR 26 \#6728.

4. Ju. I. Petunin, Conjugate Banach spaces containing subspaces of zero characteristic, Dokl. Akad. Nauk SSSR 154 (1964), 527-529 = Soviet Math. Dokl. 5 (1964), 131-133. MR 28 \#2425.

5. A. A. Pełczyński, A note on the paper of I. Singer "Basic sequences and reflexivity of Banach spaces", Studia Math. 21 (1961/62), 371-374. MR 26 \#4156.

6. I. Singer, Basic sequences and reflexivity of Banach spaces, Studia Math. 21 (1961/ 62), 351-369. MR 26 \#4155.

7. - On bases in quasi-reflexive Banach spaces, Rev. Math. Pures Appl. (Bucarest) 8 (1963), 309-311. MR 31 \#2599.

Department of Mathematics, Ohio State University, Columbus, Ohio 43210

Department of Mathematics, The Hebrew University, Jerusalem, Israel 